\title{
Cobertura de la vacunación antigripal en los farmacéuticos comunitarios: razones aportadas y absentismo relacionado. Predisposición a vacunar a la población
}

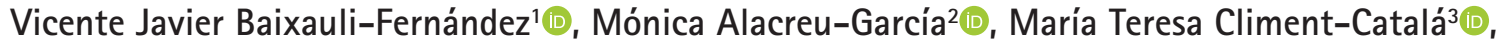 \\ Luis Salar-lbáñez ${ }^{4} \mathbb{0}$, Rosa Prats-Mas ${ }^{5} \mathbb{0}$, Cristina Aparicio-Cercós ${ }^{4} \mathbb{0}$ \\ 1. Sociedad Española de Farmacia Clínica, Familiar y Comunitaria. Farmacia comunitaria en Mislata (Valencia). 2. Departamento de Matemáticas, Física \\ y Ciencias Tecnológicas de la Universidad CEU Cardenal Herrera. Alfara del Patriarca (Valencia). 3. Sociedad Española de Farmacia Clínica, Familiar y \\ Comunitaria. Farmacia comunitaria en L'Olleria (Valencia). 4. Sociedad Española de Farmacia Clínica, Familiar y Comunitaria. Farmacia comunitaria en \\ Valencia. 5. Sociedad Española de Farmacia Clínica, Familiar y Comunitaria. Farmacia comunitaria en Denia (Alicante).
}

\section{PALABRAS CLAVE}

Vacuna contra la gripe, farmacéuticos, farmacia comunitaria, cobertura vacunal, absentismo, personal de salud, gripe humana

\section{ABREVIATURAS}

AP: Atención Primaria

FC: farmacéuticos comunitarios OMS: Organización Mundial de la Salud

SNS: Sistema Nacional de Salud VCG: vacunación contra la gripe

\section{RESUMEN}

Introducción: la vacuna antigripal está indicada entre el personal sanitario, aunque la cobertura antigripal en farmacéuticos comunitarios españoles actualmente se desconoce. Este trabajo pretende averiguar esta cobertura, las causas de los farmacéuticos para vacunarse o no de la gripe, el absentismo laboral que les ocasiona, si recomiendan la vacunación antigripal entre los grupos de riesgo y su disposición a administrarla en la farmacia.

Métodos: estudio descriptivo transversal mediante un cuestionario online destinado a farmacéuticos comunitarios de toda España. Se realizó un análisis descriptivo de las variables estudiadas y de la asociación entre la vacunación y las variables cualitativas (test Chi-cuadrado y Fisher) y cuantitativas (ANOVA).

Resultados: se obtuvo una tasa de respuesta del 9,4\% ( $\mathrm{n}=1.436)$. Los farmacéuticos refirieron vacunarse en torno al $30 \%$ en las tres temporadas estudiadas (20162019), siendo del 31-35,8 \% (IC95\%) en la temporada 2018-2019. Ser titular/cotitular de la farmacia, la experiencia profesional, la edad, vacunarse para evitar la gripe y/o por responsabilidad, y vacunarse en temporadas anteriores son factores asociados a vacunarse $(p<0,05)$. Ser socio de SEFAC también lo es. El absentismo por gripe en la temporada 2018-2019 fue del 9,5\%. Los farmacéuticos que más dijeron vacunarse recomiendan más la vacuna que los que no, y tendrían mayor predisposición a administrarla $(\mathrm{p}<0,05)$.

Conclusiones: la cobertura vacunal antigripal entre los farmacéuticos comunitarios es baja a pesar del absentismo que les causa. Facilitar la vacunación a los farmacéuticos podría incrementar su cobertura. Los farmacéuticos recomiendan la vacunación contra la gripe a los pacientes de riesgo y estarían dispuestos a vacunar en farmacias autorizadas.

\section{Introducción}

Actualmente la gripe es la enfermedad inmunoprevenible más habitual en los países desarrollados. Su gran impacto social la convierte en un auténtico problema de salud pública. La medida de control considerada más eficaz y recomendada por todas las organizaciones e instituciones nacionales y supranacionales es la vacunación anual de los grupos de población con el riesgo más alto de padecer complicaciones asociadas a esta (1).
Recibido: $21 / 9 / 2020$ Aceptado: 24/3/2021 Disponible online: 19/4/2021
Financiación: este estudio ha recibido financiación de la Sociedad Española de Farmacia Clinica, Familiar y Comunitaria (SEFAC). La financiación consistió en proporcionar sin coste a los autores de este manuscrito:

1. La programación de la encuesta web, realizada por los autores de este manuscrito.

2. El envío del correo electrónico -proporcionado por los autores de este manuscrito- a los farmacéuticos comunitarios que figuraban en su base de datos "Campus SEFAC".

3. Las respuestas obtenidas.

Conflicto de intereses: ninguno.

Cite este artículo como: Baixauli-Fernández VJ, Alacreu-Garcia M, Climent-Catalá MT, Salar-Ibáñez L, Prats-Mas R, Aparicio-Cercós C. Cobertura de la vacunación antigripal en los farmacéuticos comunitarios: razones aportadas y absentismo relacionado. Predisposición a vacunar a la población. Farmacéuticos Comunitarios. 2021 Apr 19; 13 (2): 25-35. doi:10.33620/FC.2173-9218.(2021/Nol13).002.05

Correspondencia: Vicente Javier Baixauli Fernández (vtejbaixauli@sefac.org).

ISSN 1885-8619 @SEFAC (Sociedad Española de Farmacia Clínica, Familiar y Comunitaria). Todos los derechos reservados. 
En España, la farmacia comunitaria es un establecimiento sanitario privado de interés público que lleva a cabo mayoritariamente la prestación farmacéutica del Sistema Nacional de Salud (SNS). Se estima que las 22.102 farmacias españolas atienden diariamente a más de 2,3 millones de personas siendo a menudo, por su accesibilidad y cobertura, la puerta de entrada a la Atención Primaria (AP) del sistema sanitario. Las personas que acuden a las farmacias solicitando la dispensación de algún medicamento, y en particular, la indicación de un tratamiento sintomático para la gripe, podrían ser portadores del virus, y transmitirla, tanto a su personal como a otras personas con las que allí coincidan que pueden padecer un alto riesgo de sufrir complicaciones por ésta. A su vez, si el personal de la farmacia constituido por los farmacéuticos comunitarios (FC) (2), $\mathrm{y}$ el resto de trabajadores sanitarios, es portador, puede transmitirla a las personas sanas y enfermas que acuden a ésta. Por esta razón el personal de la farmacia se encuentra incluido entre los grupos de población diana de vacunación antigripal en las recomendaciones de vacunación contra la gripe (VCG) que cada temporada publica el Consejo interterritorial del SNS (3).

Los FC, como se ha evidenciado en la reciente pandemia de COVID-19, proporcionan servicios esenciales a la comunidad, por lo que no se debe obviar el problema sanitario que supondría la disrupción de su actividad en caso de epidemia de gripe. A pesar de ello, en España se desconoce tanto el efecto de la gripe sobre el absentismo laboral de los FC, como la tasa de vacunación frente a la gripe entre éstos $\mathrm{y}$ el resto del personal de las farmacias, ya que los datos publicados por el Ministerio de Sanidad sobre cobertura vacunal entre el personal sanitario no incluyen a los FC ni a su personal. Tampoco se conocen los motivos tanto para vacunarse como para no vacunarse, lo que podría ser de utilidad para el diseño de estrategias que incrementen dichas coberturas en este colectivo. Por otro lado, la cobertura vacunal frente a la gripe continúa siendo inaceptable en nuestro país, incluso entre los grupos de riesgo $(4,5)$, ya que sigue sin alcanzarse el objetivo del Ministerio de Sanidad de alcanzar coberturas vacunales cercanas a los objetivos establecidos por la Organización Mundial de la Salud (OMS) y por la Comisión Europea (3). Por ello, algunas estrategias dirigidas a aumentar esta cobertura apuntan hacia una participación más activa de los profesionales sanitarios y a vacunar contra la gripe en farmacias autorizadas $(5,6)$. En este sentido, se desconoce la disposición de los FC a desempeñar un papel más activo de acuerdo con estas estrategias.

\section{Objetivos}

Los objetivos del estudio fueron conocer entre los FC la tasa de vacunación en las temporadas antigripales 2016 a 2018, describir las razones que inducen a los FC a vacunarse o no vacunarse contra la gripe, cuantificar el absentismo laboral causado por la gripe entre los FC, indagar si los FC recomiendan la VCG a los grupos de riesgo y conocer la disposición de los FC a administrar la VCG en la farmacia.

\section{Métodos}

Estudio observacional descriptivo transversal realizado mediante un cuestionario online entre FC de toda España.

La población de estudio estuvo constituida por los 53.305 FC (2) colegiados ejercientes en las farmacias españolas. De ellos, se incluyeron a $15.301 \mathrm{FC}(28,7 \%)$ registrados en la base de datos de "Campus SEFAC" propiedad de la Sociedad Española de Farmacia Clínica, Familiar y Comunitaria (SEFAC). Estar registrado en dicha base de datos no implicó ser socio de SEFAC, sino haber participado en alguna actividad formativa promovida por SEFAC. El tamaño muestral necesario para estimar el porcentaje de VCG en este colectivo, presupuesto en un 50\%, con una precisión del 3\% y un nivel de confianza del 95\%, fue de 1.045 FC.

El estudio se desarrolló en el ámbito de la farmacia comunitaria, tras un pilotaje previo y finalizada la campaña de VCG, durante un mes, del 18 de febrero al 18 de marzo de 2019. La participación de los FC en el estudio fue anónima, voluntaria y altruista.

Todas las variables se recogieron en un cuestionario diseñado para este estudio (figura 1).

Los datos sociodemográficos de los FC participantes constituyeron las variables independientes y las variables dependientes fueron: vacunación del FC en cada una de las tres temporadas: 2016-2018, los días de absentismo laboral causados por la gripe en la temporada 2018-19, las causas por las que se vacunó o no contra la gripe, si recomendó la VCG a los grupos de riesgo y si administraría la VCG en la farmacia.

Se envió un correo electrónico a 15.301 FC invitándoles a participar en el estudio. El FC pudo elegir entre participar, no participar en el estudio o bien que se le recordara esta elección hasta el 18 de marzo de 2019, fecha final para participar. Los que eligieron participar accedieron directamente y una única vez al cuestionario a cumplimentar. El cuestionario se alojó en el portal de investigación de SEFAC, bajo el software online webform desarrollado en Drupal.

Los datos obtenidos se analizaron mediante el software estadístico R. En primer lugar, se obtuvo un intervalo de confianza al 95\% (IC 95\%) para la estimación de la prevalencia de VCG en la última campaña. Para llevar a cabo la inferencia, se clasificó a los FC por adherencia a la VCG en tres perfiles según se hubieran vacunado en las tres campañas, perfil "Siempre", en una o dos de ellas, perfil "Alguna vez" o en ninguna de las tres, perfil "Nunca”. También se categorizó el tiempo de absentismo laboral en tres grupos: "0 días", "1-10 días”, ">10 días". Se investigó la asociación de la adherencia a VCG con el resto de variables: sexo, comunidad autónoma, titularidad, absentismo laboral categorizado, motivos de vacunación y de no vacunación, recomendación de la VCG, administración autorizada de la VCG (Test Chi-cuadrado y exacto de Fisher) y con la edad y el tiempo de ejercicio (ANOVA).

Para participar en el estudio todos los FC participantes otorgaron previamente su consentimiento informado. El estudio contó con el informe favorable del Comité de Ética para la Investigación Biomédica de la Universidad CEU Cardenal Herrera. 
Estimado/a compañero/a:

Desde la Sociedad Española de Farmacia Familiar y Comunitaria (SEFAC) se pretende realizar el estudio titulado: VACUNACIÓN DE LA GRIPE DE LOS FARMACÉUTICOS COMUNITARIOS ESPAÑOLES EN LAS CAMPAÑAS ANTIGRIPALES ENTRE 2016 Y 2019. El Comité de Ética para la investigación biomédica de la Universidad CEU-Cardenal Herrera lo ha revisado y acepta su realización. Dicho estudio consiste en conocer a través de la cumplimentación de una encuesta anónima de 7 preguntas, cuántos farmacéuticos comunitarios deciden vacunarse de la gripe en nuestro pais, y las causas de dicha decisión (vacunarse o no), ya que actualmente no se dispone de esta información. Además, nos gustaría conocer el absentismo laboral que provoca la gripe en este colectivo, asi como si el farmacéutico comunitario recomienda la vacunación a los pacientes incluidos en los grupos de riesgo y estaría dispuesto a administrar la vacuna antigripal en la farmacia. Los datos obtenidos servirán para conocer y analizar la realidad de la vacunación antigripal entre los farmacéuticos comunitarios españoles.

Con este fin solicito tu colaboración altruista, desinteresada y sincera.

Para ello, hemos diseñado una breve encuesta que no te llevará contestarla más de un par de minutos. Esta encuesta es totalmente anónima, y no requiere de ningún dato personal que te identifique.

\begin{tabular}{|c|}
\hline ACEPTO PARTICIPAR \\
EN EL ESTUDIO \\
\hline
\end{tabular}

Agradeciéndote de antemano tu colaboración

xxxxxx

\section{CUESTIONARIO DEL ESTUDIO}

\section{VACUNACIÓN DE LA GRIPE DE LOS FARMACÉUTICOS COMUNITARIOS ESPAÑOLES EN LAS CAMPAÑAS ANTIGRIPALES ENTRE 2016 Y 2019.}

Por favor, marca con una $\mathrm{X}$ la opción elegida

1. Perfil del farmacéutico comunitario participante:

Sexo: $\square$ HOMBRE $\square$ MUJER

Edad:___ (número entero)

Provincia donde ejerce:

¿Eres farmacéutico titular/cotitular?

sí (listado de selección)

¿Eres socio de SEFAC? $\square$ Sí $\square$ NO

Años de ejercicio en farmacia comunitaria: (número entero)

2. Indique en cuál/es campaña de vacunación se ha vacunado

$\square$ 2016-2017 $\square$ 2017-2018 $\square$ 2018-2019

3. ¿Cuántos días de absentismo laboral te ha causado la gripe en la campaña antigripal 2018-2019? (número entero)

4. ¿Por qué se vacuna? (Marcar con una $X$ la causa/s elegidas):

O Porque pertenezco a un grupo de riesgo

O Por prevención, para no padecer la gripe

O Por responsabilidad, así aumento la cobertura vacunal

O Porque me lo ha recomendado el médico u otro profesional sanitario

Otras (detallar):

5. ¿Por qué no se vacuna? (Marcar con una $\mathrm{X}$ la causa/s elegidas):

O No me lo han recomendado nunca

O No es necesario vacunarme, no pertenezco a un grupo de riesgo

O Confianza en la seguridad de las vacunas: me puede provocar alguna reacción no deseada o la tengo contraindicada

O Confianza en la eficacia de las vacunas: no me fío de la vacuna, no tienen el efecto esperado

O Comodidad para la vacunación: no resulta fácil vacunarme (tener receta, comprar en la farmacia, pedir cita, ir al centro de salud a que te vacunen, falta de tiempo,...)

O Olvido: se me ha pasado

O Otras (detallar):

6. ¿Recomienda la vacunación a los pacientes incluidos en los grupos de riesgo que acuden a la farmacia? $\quad \square$ Sí $\quad \square$ NO

7. ¿Estaría dispuesto a administrar la vacuna de la gripe en la farmacia si la administración sanitaria lo autorizase? $\quad \square$ Sí $\quad \square$ NO 


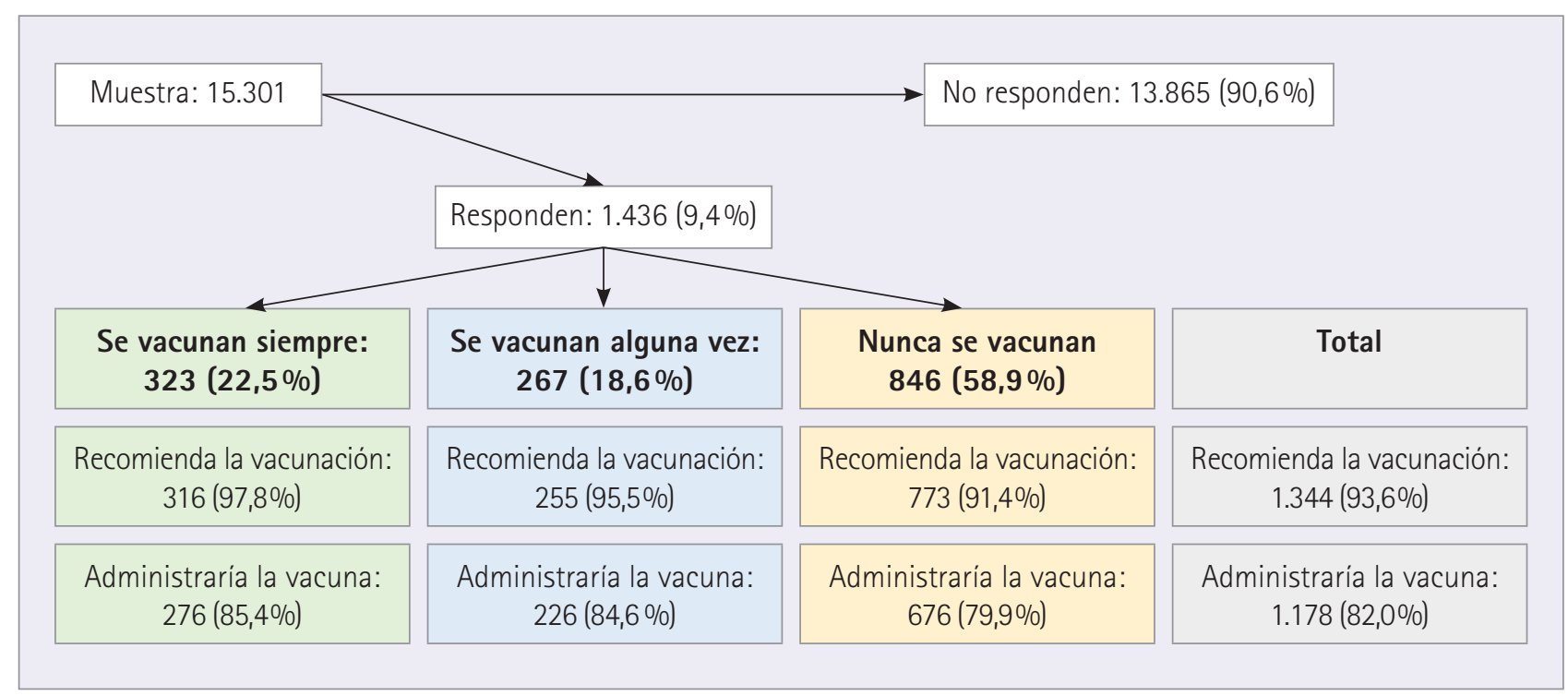

Figura 2 Esquema general del estudio

\section{Resultados}

De los 15.301 FC accesibles, el $9,4 \%(n=1.436)$ aceptó participar en el estudio superándose el tamaño muestral necesario. Un 0,03\% (5) no participaron, y el resto no contestó. En la figura 2 puede verse un resumen de los resultados más importantes.

Las cifras de vacunación referidas en las tres campañas investigadas fueron: 441 (30,7\%) en 2016-2017, $423(29,5 \%)$ en 2017-2018 y 479 $(33,4 \%)$ en 2018-2019. Existe relación significativa entre vacunarse en la última campaña y haberse vacunado en alguna de las campañas anteriores $(p<0.05)$. Se estima que en esta última campaña se vacunaron entre el 30,9 y el 35,8\% (IC 95\%) de los FC.
Para analizar las diferencias entre los FC éstos se agruparon en tres perfiles de adherencia a la VCG: perfil "Siempre” (22,5\%), "Alguna vez" (18,6\%) y "Nunca" (58,9\%) (figura 3). Como se observa en la tabla 1, el 76,5\% de los participantes son mujeres, distribución que se mantiene aproximadamente en los tres perfiles de FC ( $>>0,05)$. Los socios de SEFAC tuvieron una mayor adherencia a la VCG que los que no lo son $(p<0,05)$. El 25,3\% de los socios de SEFAC se vacuna siempre, frente al $17,5 \%$ de los no socios, y el 54,9\% de los socios de SEFAC nunca se vacuna frente al 66,0\% de los no socios.

El 52,2\% de los FC participantes son titulares/cotitulares de farmacias, siendo ésta una variable asociada significativamente a la adherencia a la

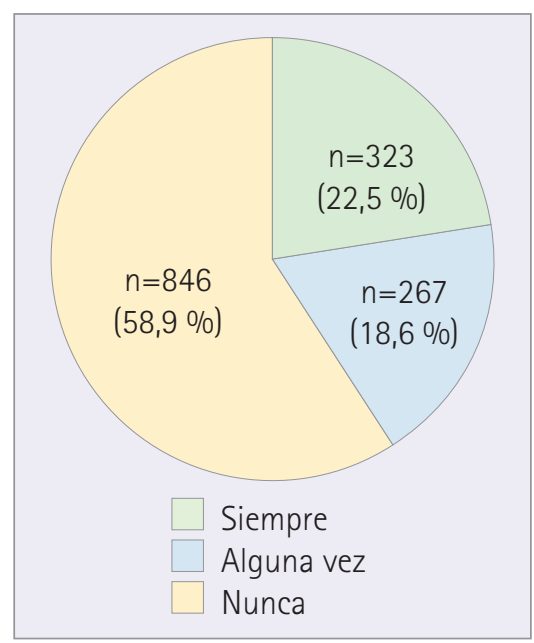

Figura 3 Farmacéuticos comunitarios que refieren estar vacunados contra la gripe en las tres temporadas analizadas: 2016-2017, 2017-2018 y 2018-2019

Tabla 1 Asociación entre la frecuencia de vacunación contra la gripe y las caracteristicas sociodemográficas

\begin{tabular}{|c|c|c|c|c|c|}
\hline & \multirow[b]{2}{*}{$\begin{array}{c}\text { Totales } \\
\text { n (\%) }\end{array}$} & \multicolumn{3}{|c|}{ Farmacéuticos vacunados } & \multirow[b]{2}{*}{$\begin{array}{l}\text { p-valor } \\
\text { Test } \chi^{2}\end{array}$} \\
\hline & & $\begin{array}{l}\text { Siempre } \\
\text { n (\%) }\end{array}$ & $\begin{array}{c}\text { Alguna vez } \\
\text { n (\%) }\end{array}$ & $\begin{array}{l}\text { Nunca } \\
\text { n (\%) }\end{array}$ & \\
\hline $\begin{array}{l}\text { Sexo } \\
\text { Hombre } \\
\text { Mujer }\end{array}$ & $\begin{array}{c}337(23,5 \%) \\
1099(76,5 \%)\end{array}$ & $\begin{array}{c}85(26,3 \%) \\
238(73,7 \%)\end{array}$ & $\begin{array}{c}72(27 \%) \\
195(73 \%)\end{array}$ & $\begin{array}{l}180(21,3 \%) \\
666(78,7 \%)\end{array}$ & 0,06267 \\
\hline $\begin{array}{l}\text { Socio SEFAC } \\
\text { NO } \\
\text { sí }\end{array}$ & $\begin{array}{l}515(35,9 \%) \\
921(64,1 \%)\end{array}$ & $\begin{array}{c}90(27,9 \%) \\
233(72,1 \%)\end{array}$ & $\begin{array}{c}85(31,8 \%) \\
182(68,2 \%)\end{array}$ & $\begin{array}{l}340(40,2 \%) \\
506(59,8 \%)\end{array}$ & $1,397 e^{-4}$ \\
\hline $\begin{array}{l}\text { Titular/Cotitular } \\
\text { NO } \\
\text { sí }\end{array}$ & $\begin{array}{l}687(47,8 \%) \\
749(52,2 \%)\end{array}$ & $\begin{array}{l}110(34,1 \%) \\
213(65,9 \%)\end{array}$ & $\begin{array}{l}121(45,3 \%) \\
146(54,7 \%)\end{array}$ & $\begin{array}{l}456(53,9 \%) \\
390(46,1 \%)\end{array}$ & $6,418 e^{-9}$ \\
\hline Totales & $1.436(100 \%)$ & $323(100 \%)$ & $267(100 \%)$ & $846(100 \%)$ & \\
\hline
\end{tabular}




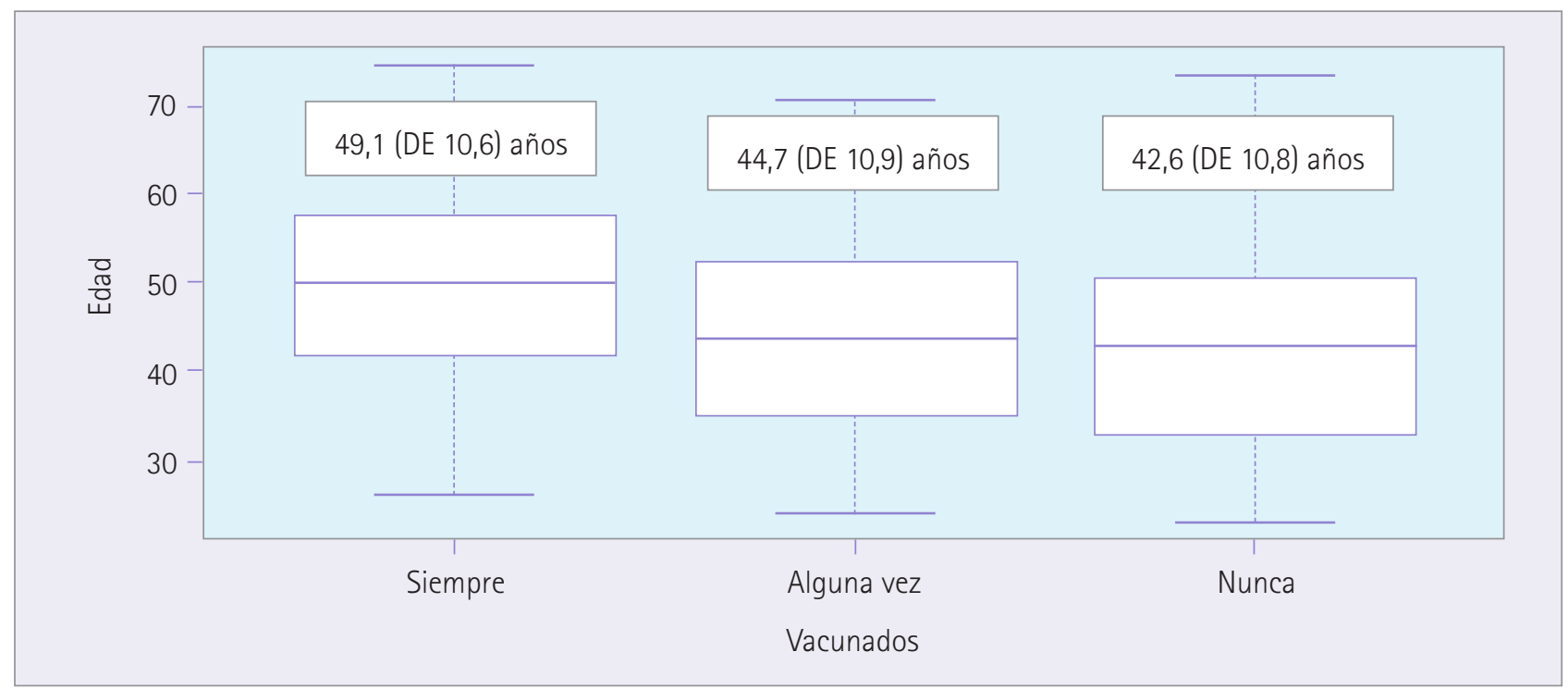

Figura 4 Frecuencia de vacunación contra la gripe según la edad de los farmacéuticos comunitarios participantes en el estudio

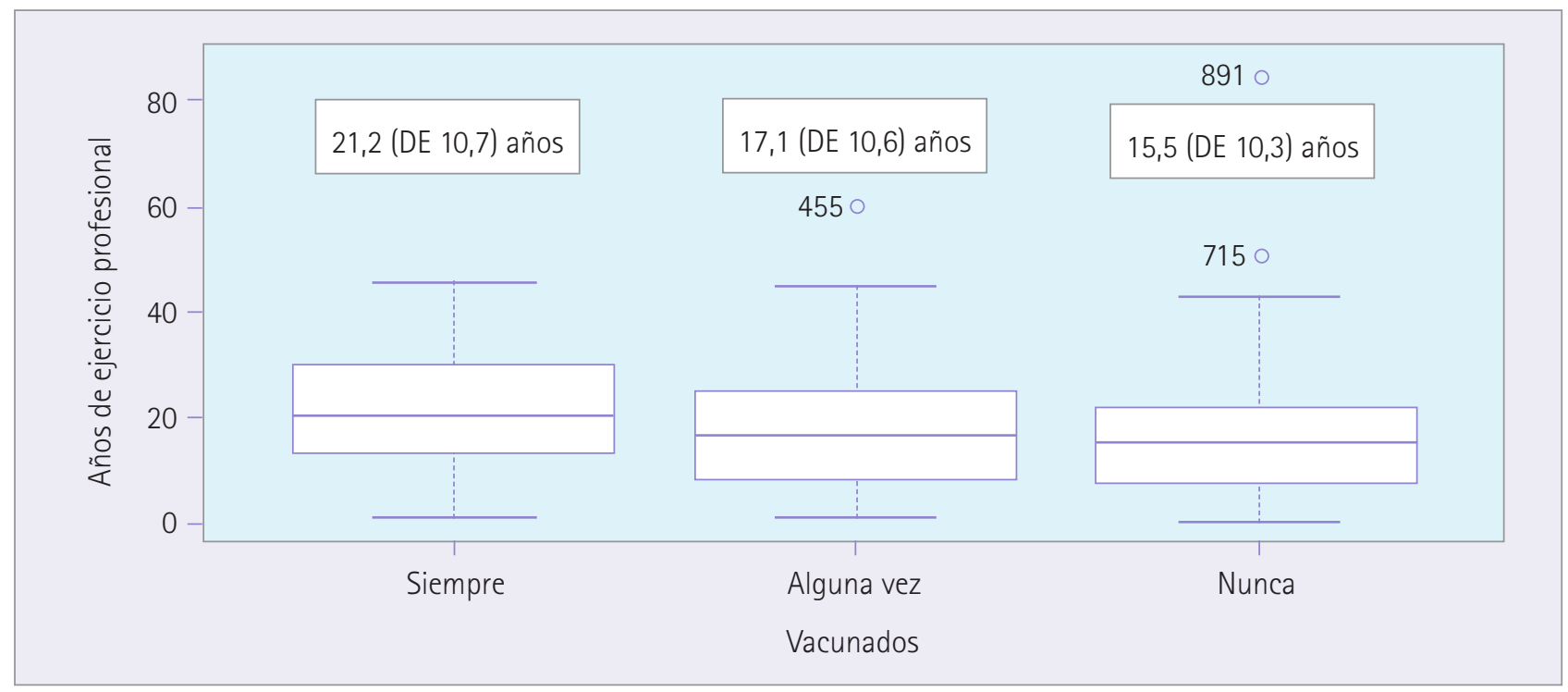

Figura 5 Frecuencia de vacunación contra la gripe según los años de ejercicio profesional de los farmacéuticos comunitarios participantes en el estudio

VCG $(\mathrm{p}<0,05)$. En la figura 4 se puede observar que la edad de los FC influyó significativamente $(\mathrm{p}<0,05)$ en la concienciación sobre la VCG en sentido positivo. Así mismo (figura 5), el tiempo medio de ejercicio profesional fue significativamente mayor en FC que se vacunan "Siempre" respecto al resto. La pertenencia a una comunidad autónoma no influyó significativamente sobre la VCG de los FC (tabla 3).

El 90,6\% de los FC no se ausentaron por la gripe en la campaña 2018-2019, el 8,9\% se ausentó entre 1-10 días y el 0,5\% más de 10 días. Según los resultados de la tabla 2 , el absentismo laboral por gripe no se asocia con haberse vacunado o no. El tiempo medio de absentismo laboral tampoco está relacionado con ninguna otra variable, excepto con la edad de los FC, con la que presenta una asociación lineal positiva (Test de correlación de Pearson $\mathrm{p}=0,01925$ ).

La tabla 4 muestra las causas de los FC para vacunarse, ordenados de mayor a menor frecuencia. El porcentaje de FC que se vacunaron para evitar la gripe y/o por responsabilidad fue significativamente superior entre los FC "Siempre" que entre los FC "Alguna vez". En el resto de motivos no hay diferencias significativas entre los FC "Siempre" y "Alguna vez". La tabla 5 muestra separada y conjuntamente los motivos por los que los FC "Alguna vez" y "Nunca" no se vacunan.

Según los resultados de la tabla 6, un 93,6\% de los FC recomienda la VCG a los grupos de riesgo y un $82 \%$ estaría dispuesto a administrarla en la farmacia si Sanidad lo autorizase, siendo estadísticamente significativo $(\mathrm{p}<0,05)$ que los $\mathrm{FC}$ que más se vacunan recomiendan más la VCG que los que no se vacunan y tendrían mayor predisposición a administrar la VCG. 
Tabla 2 Asociación entre el absentismo laboral y haber recibido o no la vacuna contra la gripe, en la campaña 2018 - 2019

\begin{tabular}{|c|c|c|c|c|c|}
\hline \multirow[b]{2}{*}{$\begin{array}{l}\text { Vacunados en } \\
\text { 2018-2019 }\end{array}$} & \multirow[b]{2}{*}{ Totales } & \multicolumn{3}{|c|}{ Absentismo laboral } & \multirow{2}{*}{$\begin{array}{c}\text { p-valor } \\
\text { Test exacto de } \\
\text { Fisher }\end{array}$} \\
\hline & & $\begin{array}{l}0 \text { días } \\
\text { n (\%) }\end{array}$ & $\begin{array}{c}1-10 \text { días } \\
n(\%)\end{array}$ & $\begin{array}{c}>10 \text { dias } \\
\text { n (\%) }\end{array}$ & \\
\hline $\begin{array}{l}\text { NO } \\
\text { sí }\end{array}$ & $\begin{array}{l}957(66,6 \%) \\
479(33,4 \%)\end{array}$ & $\begin{array}{l}865(66,5 \%) \\
435(33,5 \%)\end{array}$ & $\begin{array}{l}86(67,2 \%) \\
42(32,8 \%)\end{array}$ & $\begin{array}{l}6(75 \%) \\
2(25 \%)\end{array}$ & 0,936 \\
\hline Totales & $1.436(100 \%)$ & $1.300(100 \%)$ & $128(100 \%)$ & $8(100 \%)$ & \\
\hline
\end{tabular}

Tabla 3 Distribución de los participantes por comunidades, según se han vacunado o no contra la gripe en la campaña 2018-2019 y predisposición a administrar la vacuna contra la gripe, si se autorizara

\begin{tabular}{|c|c|c|c|c|c|}
\hline \multirow[b]{2}{*}{ Comunidades Autónomas } & \multirow[b]{2}{*}{$\begin{array}{c}\text { Totales } \\
\text { n (\%) }\end{array}$} & \multicolumn{2}{|c|}{ Vacunados en 2018-2019 } & \multicolumn{2}{|c|}{ Administrarías la vacuna } \\
\hline & & $\begin{array}{c}\text { NO } \\
\text { n (\%) }\end{array}$ & $\begin{array}{c}\text { sí } \\
\text { n (\%) }\end{array}$ & $\begin{array}{c}\text { NO } \\
\text { n (\%) }\end{array}$ & $\begin{array}{c}\text { si } \\
\text { n (\%) }\end{array}$ \\
\hline Andalucía & $194(13,5 \%)$ & $132(13,8 \%)$ & $62(12,9 \%)$ & $28(10,9 \%)$ & $166(14,1 \%)$ \\
\hline Aragón & $66(4,6 \%)$ & $42(4,4 \%)$ & $24(5 \%)$ & $20(7,8 \%)$ & $46(3,9 \%)$ \\
\hline Comunidad de Madrid & $194(13,5 \%)$ & $142(14,8 \%)$ & $52(10,9 \%)$ & $21(8,1 \%)$ & $173(14,7 \%)$ \\
\hline Cantabria & $16(1,1 \%)$ & $11(1,1 \%)$ & $5(1 \%)$ & $3(1,2 \%)$ & $13(1,1 \%)$ \\
\hline Castilla-La Mancha & $79(5,5 \%)$ & $51(5,3 \%)$ & $28(5,8 \%)$ & $14(5,4 \%)$ & $65(5,5 \%)$ \\
\hline Castilla y León & $59(4,1 \%)$ & $36(3,8 \%)$ & $23(4,8 \%)$ & $13(5 \%)$ & $46(3,9 \%)$ \\
\hline Cataluña & $117(8,2 \%)$ & $73(7,6 \%)$ & $44(9,2 \%)$ & $15(5,8 \%)$ & $102(8,7 \%)$ \\
\hline Comunidad Valenciana & $202(14,1 \%)$ & $128(13,4 \%)$ & $74(15,4 \%)$ & $26(10,1 \%)$ & $176(14,9 \%)$ \\
\hline Extremadura & $34(2,4 \%)$ & $24(2,5 \%)$ & $10(2,1 \%)$ & $10(3,9 \%)$ & $24(2 \%)$ \\
\hline Galicia & $151(10,5 \%)$ & $102(10,7 \%)$ & $49(10,2 \%)$ & $34(13,2 \%)$ & $117(9,9 \%)$ \\
\hline Islas Baleares & $50(3,5 \%)$ & $34(3,6 \%)$ & $16(3,3 \%)$ & $15(5,8 \%)$ & $35(3 \%)$ \\
\hline Canarias & $61(4,3 \%)$ & $39(4,1 \%)$ & $22(4,6 \%)$ & $17(6,6 \%)$ & $44(3,7 \%)$ \\
\hline La Rioja & $8(0,6 \%)$ & $5(0,5 \%)$ & $3(0,6 \%)$ & $2(0,8 \%)$ & $6(0,5 \%)$ \\
\hline Melilla & $1(0,1 \%)$ & $1(0,1 \%)$ & $0(0 \%)$ & $0(0 \%)$ & $1(0,1 \%)$ \\
\hline Navarra & $18(1,3 \%)$ & $12(1,3 \%)$ & $6(1,3 \%)$ & $3(1,2 \%)$ & $15(1,3 \%)$ \\
\hline Principado de Asturias & $45(3,1 \%)$ & $32(3,3 \%)$ & $13(2,7 \%)$ & $9(3,5 \%)$ & $36(3,1 \%)$ \\
\hline País Vasco & $81(5,6 \%)$ & $54(5,6 \%)$ & $27(5,6 \%)$ & $17(6,6 \%)$ & $64(5,4 \%)$ \\
\hline Región de Murcia & $60(4,2 \%)$ & $39(4,1 \%)$ & $21(4,4 \%)$ & $11(4,3 \%)$ & $49(4,2 \%)$ \\
\hline Totales & $1.436(100 \%)$ & 957 (100\%) & 479 (100\%) & $258(100 \%)$ & $1.178(100 \%)$ \\
\hline
\end{tabular}


Tabla 4 Asociación entre la frecuencia de vacunación contra la gripe y los motivos de vacunación

\begin{tabular}{|c|c|c|c|c|}
\hline \multirow[b]{2}{*}{ Motivos de vacunación } & \multirow[b]{2}{*}{$\begin{array}{c}\text { Totales } \\
\text { n (\%) }\end{array}$} & \multicolumn{2}{|c|}{ Farmacéuticos vacunados } & \multirow[b]{2}{*}{$\begin{array}{l}\text { p-valor } \\
\text { Test } \chi^{2}\end{array}$} \\
\hline & & $\begin{array}{c}\text { Siempre } \\
n(\%)\end{array}$ & $\begin{array}{c}\text { Alguna vez } \\
\text { n (\%) }\end{array}$ & \\
\hline $\begin{array}{l}\text { Pertenencia a grupo de riesgo } \\
\text { NO } \\
\text { Sí }\end{array}$ & $\begin{array}{l}217(36,8 \%) \\
373(63,2 \%)\end{array}$ & $\begin{array}{l}118(36,5 \%) \\
205(63,7 \%)\end{array}$ & $\begin{array}{c}99(37,1 \%) \\
168(62,9 \%)\end{array}$ & 0,8911 \\
\hline $\begin{array}{l}\text { Evitar la gripe } \\
\text { NO } \\
\text { Sí }\end{array}$ & $\begin{array}{l}292(49,5 \%) \\
298(50,5 \%)\end{array}$ & $\begin{array}{l}140(43,3 \%) \\
183(56,7 \%)\end{array}$ & $\begin{array}{l}152(56,9 \%) \\
115(43,1 \%)\end{array}$ & 0,001 \\
\hline $\begin{array}{l}\text { Por responsabilidad } \\
\text { NO } \\
\text { sí }\end{array}$ & $\begin{array}{l}333(56,4 \%) \\
257(43,6 \%)\end{array}$ & $\begin{array}{l}164(50,8 \%) \\
159(49,2 \%)\end{array}$ & $\begin{array}{c}169(63,3 \%) \\
98(36,7 \%)\end{array}$ & 0,0023 \\
\hline $\begin{array}{l}\text { Por recomendación médica } \\
\text { NO } \\
\text { sí }\end{array}$ & $\begin{array}{c}542(91,9 \%) \\
48(8,1 \%)\end{array}$ & $\begin{array}{c}302(93,5 \%) \\
21(6,5 \%)\end{array}$ & $\begin{array}{c}240(89,9 \%) \\
27(10,1 \%)\end{array}$ & 0,1103 \\
\hline $\begin{array}{l}\text { Otros } \\
\text { NO } \\
\text { sí }\end{array}$ & $\begin{array}{c}527(89,3 \%) \\
63(10,7 \%)\end{array}$ & $\begin{array}{c}292(90,4 \%) \\
31(9,6 \%)\end{array}$ & $\begin{array}{l}235(88 \%) \\
32(12 \%)\end{array}$ & 0,35 \\
\hline Totales & $590(100 \%)$ & $323(100 \%)$ & $267(100 \%)$ & \\
\hline
\end{tabular}

Tabla 5 Asociación entre la frecuencia de vacunación contra la gripe y los motivos de no vacunación contra la gripe

\begin{tabular}{|c|c|c|c|c|}
\hline \multirow[b]{2}{*}{ Motivos de no vacunación } & \multirow[b]{2}{*}{$\begin{array}{l}\text { Totales } \\
\text { n (\%) }\end{array}$} & \multicolumn{2}{|c|}{ Farmacéuticos vacunados } & \multirow{2}{*}{$\begin{array}{c}\text { p-valor } \\
\text { Test exacto de } \\
\text { Fisher }\end{array}$} \\
\hline & & $\begin{array}{c}\text { Alguna vez } \\
\text { n (\%) }\end{array}$ & $\begin{array}{l}\text { Nunca } \\
\mathrm{n}(\%)\end{array}$ & \\
\hline $\begin{array}{l}\text { No me lo han recomendado } \\
\text { No } \\
\text { sí }\end{array}$ & $\begin{array}{l}904(81,2 \%) \\
209(18,8 \%)\end{array}$ & $\begin{array}{c}246(92,1 \%) \\
21(7,9 \%)\end{array}$ & $\begin{array}{l}658(77,8 \%) \\
188(22,2 \%)\end{array}$ & $2,881 e^{-8}$ \\
\hline $\begin{array}{l}\text { No pertenezco a un grupo de riesgo } \\
\text { NO } \\
\text { sí }\end{array}$ & $\begin{array}{l}917(82,4 \%) \\
196(17,6 \%)\end{array}$ & $\begin{array}{c}251(94 \%) \\
16(6 \%)\end{array}$ & $\begin{array}{l}666(78,7 \%) \\
180(21,3 \%)\end{array}$ & $6,574 \mathrm{e}^{-10}$ \\
\hline $\begin{array}{l}\text { Porque no son seguras } \\
\text { NO } \\
\text { sí }\end{array}$ & $\begin{array}{c}1.070(96,1 \%) \\
43(3,9 \%)\end{array}$ & $\begin{array}{c}260(97,4 \%) \\
7(2,6 \%)\end{array}$ & $\begin{array}{c}810(95,7 \%) \\
36(4,3 \%)\end{array}$ & 0,2765 \\
\hline $\begin{array}{l}\text { Porque no son efectivas } \\
\text { no } \\
\text { sí }\end{array}$ & $\begin{array}{c}1.041(93,5 \%) \\
72(6,5 \%)\end{array}$ & $\begin{array}{c}261(97,8 \%) \\
6(2,2 \%)\end{array}$ & $\begin{array}{c}780(92,2 \%) \\
66(7,8 \%)\end{array}$ & $8,556 \mathrm{e}^{-4}$ \\
\hline $\begin{array}{l}\text { Falta de comodidad } \\
\text { NO } \\
\text { sí }\end{array}$ & $\begin{array}{l}835(75 \%) \\
278(25 \%)\end{array}$ & $\begin{array}{c}205(76,8 \%) \\
62(23,2 \%)\end{array}$ & $\begin{array}{l}630(74,5 \%) \\
216(25,5 \%)\end{array}$ & 0,4666 \\
\hline $\begin{array}{l}\text { Se me olvidó } \\
\text { NO } \\
\text { sí }\end{array}$ & $\begin{array}{l}815(73,2 \%) \\
298(26,8 \%)\end{array}$ & $\begin{array}{l}130(48,7 \%) \\
137(51,3 \%)\end{array}$ & $\begin{array}{l}685(81 \%) \\
161(19 \%)\end{array}$ & 0 \\
\hline $\begin{array}{l}\text { Otros } \\
\text { NO } \\
\text { sí }\end{array}$ & $\begin{array}{l}938(84,3 \%) \\
175(15,7 \%)\end{array}$ & $\begin{array}{c}231(86,5 \%) \\
36(13,5 \%)\end{array}$ & $\begin{array}{l}707(83,6 \%) \\
139(16,4 \%)\end{array}$ & 0,2488 \\
\hline Totales & $1.113(100 \%)$ & $267(100 \%)$ & $846(100 \%)$ & \\
\hline
\end{tabular}


Tabla 6 Asociación entre la frecuencia de vacunación contra la gripe, la recomendación de la vacuna contra la gripe a los grupos de riesgo y la predisposición a administrar la vacuna contra la gripe, si se autorizase

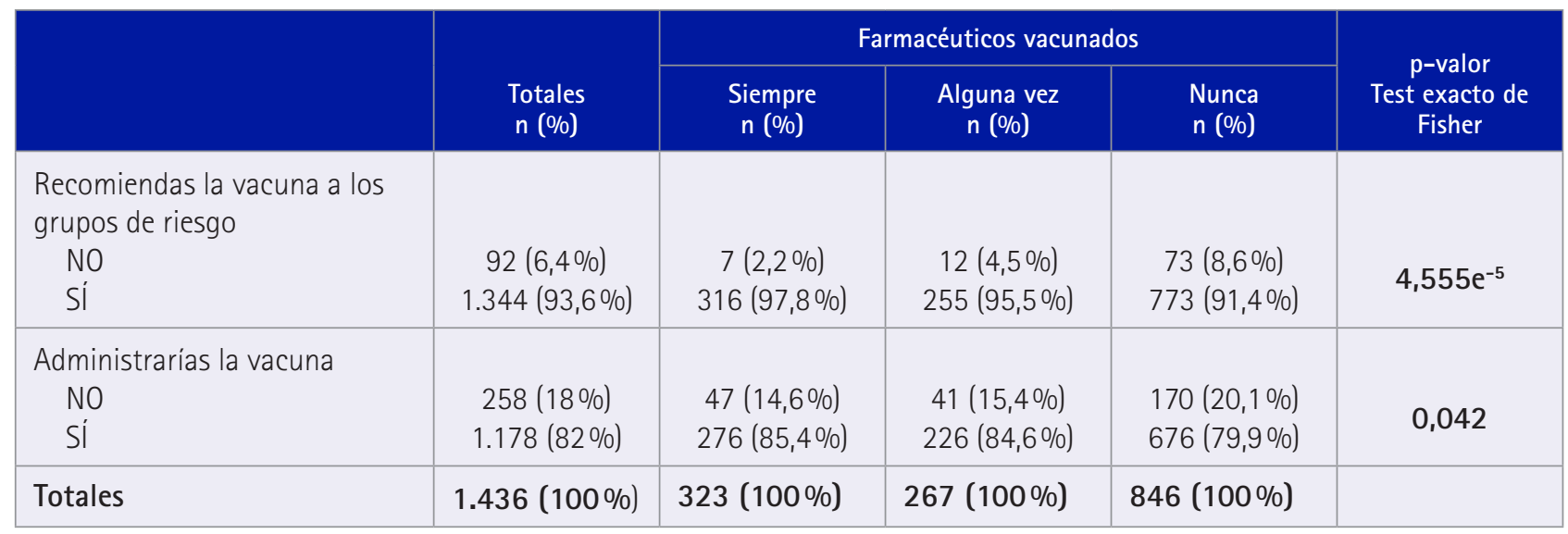

\section{Discusión}

Habitualmente la participación en los cuestionarios online suele ser muy baja. La tasa de respuesta obtenida en este estudio $(9,4 \%)$ ha sido baja, pero podemos considerarla dentro de lo esperable, más aún, teniendo en cuenta que se hizo al principio del confinamiento por la pandemia de COVID-19, si bien ha sido ligeramente mayor que la obtenida en el único estudio con características similares realizado con FC catalanes (7,3\%) (7). En otro estudio realizado con todos los trabajadores de las farmacias de Lleida la participación fue del 43,1\%, si bien para alcanzarla hubo que realizar además entrevistas telefónicas y presenciales debido a la baja participación de algunas farmacias (8).

En España no se conocen bien las coberturas de VCG en el personal sanitario (9), pero todos los estudios publicados sobre la cobertura vacunal frente a la gripe de los trabajadores sanitarios coinciden en que ésta es baja y sigue muy alejada del 75\% que recomienda la OMS y presentan otros países occidentales. Las tasas de VCG obtenidas en las temporadas estudiadas: 30,7\% (2016-2017), 29,5\% (2017-2018) y 33,4\% (2018-2019) son muy similares a las publicadas por el Ministerio de Sanidad para los profesionales sanitarios en dichas temporadas $(31,5 \%, 31,3$ y 33,9\%, respectivamente) $(4,10,11)$. Estos estudios han sido realizados casi exclusivamente en centros hospitalarios, sociosanitarios y de AP, encontrándose sólo dos $(7,8)$ en farmacias comunitarias, los dos en Cataluña. La tasa de VCG de 2018-2019 entre los FC (33,4\%) es ligeramente inferior a la obtenida $(39,8 \%)$ en un estudio realizado mediante 800 entrevistas a médicos y enfermeros de centros de AP de quince comunidades autónomas (11). Esta diferencia podría deberse a la mayor facilidad que tienen estos últimos para vacunarse. Si se compara con las coberturas de los FC catalanes que datan de la temporada 2013-2014 y 2010-2011, estas son sensiblemente menores: $25,1 \%$ (7) y 19,8\% (8), respectivamente y teniendo en cuenta que ésta última incluyó también al resto del personal de las farmacias participantes. Aun así, esta tasa es muy inferior a la referida por los FC estadounidenses en 2008, que fue del $75 \%$ (12).

La pertenencia a una sociedad científica, el ser FC titular/cotitular de la farmacia, la experiencia profesional y la edad, son factores que se asociaron de forma estadísticamente significativa a vacunarse. Esto podría explicarse por la mayor responsabilidad profesional que conllevan todas estas variables. La diferencia entre FC titulares y no titulares podría ser debida a que los segundos han de pedir permiso a los primeros para ir a que les administren la vacuna antigripal. En cuanto a la experiencia profesional, los más experimentados son más mayores y, probablemente, la mayor edad aumente la intención de prevenir la salud o de sufrir alguna enfermedad crónica o factor de riesgo relacionado con la gripe, que haga que se vacunen más. Los resultados de otros estudios sobre FC (7), trabajadores de la farmacia (8) y centros sanitarios españoles también arrojan diferencias significativas entre categorías profesionales de médicos (pediatras y otras especialidades), personal de enfermería (enfermeros y auxiliares de enfermería) y personal administrativo (13-16), así como con el aumento de la edad (7,8,14-17). En este estudio el sexo no explicó la adherencia a la VCG $(p>0,05)$ y en otros estudios de otros profesionales sanitarios $(15,17)$ se encontraron resultados dispares, por lo que no parecen existir diferencias significativas entre hombres y mujeres (13). Como en otros estudios sobre FC $(7,8)$, también se encontró relación estadísticamente significativa $(\mathrm{p}<0,05)$ entre vacunarse en la última temporada y haberse vacunado en alguna de las temporadas anteriores.

\section{Causas}

Son varios los estudios previos que han intentado conocer los motivos por los que el personal sanitario se vacuna contra la gripe y las razones para no hacerlo. Las tres causas más frecuentes referidas para vacunarse contra la gripe en éste y otros estudios $(7,8)$, tanto por los FC como por los profesionales sanitarios de centros de salud de AP $(14,17,19,20)$ y centros hospitalarios (16) coinciden con las tres razones que hacen incuestionable su recomendación en el personal sanitario: el argumento de necesidad (es una medida de autoprotección, puesto que el profesional sanitario tiene más oportunidades de resultar infectado por el virus de la gripe que la población general), el argumento de ética (de no hacer daño al paciente, ya que el 
trabajador sanitario no debe ser causa de enfermedades evitables en los pacientes a su cuidado, ni a sus compañeros), y el argumento de ejemplaridad (el profesional que se vacune tendrá mayor concienciación sobre las ventajas de la vacunación y será más proclive a recomendarla en los grupos de riesgo) $(6,18,19)$.

Sin embargo, la mayoría de las causas por las que los FC refieren no vacunarse, están relacionadas con aspectos prácticos: "olvido", "falta de comodidad", y "no he recibido la recomendación". Las tres se podrían subsanar implementando medidas que facilitasen, recordasen y recomendasen la VCG a este colectivo, organizando su vacunación al igual que ocurre con los trabajadores sanitarios del SNS. Sólo un estudio de trabajadores sanitarios de un área de $\mathrm{AP}$ situó en el 19\% la "pereza o dejadez" como motivo más frecuente para no vacunarse (21).

Sólo una minoría de los FC que refieren no vacunarse alegan los mismos motivos que, sin embargo, afirman mayoritariamente el resto de profesionales y trabajadores sanitarios para no vacunarse $(7,8,19,20-22)$, estando relacionados con actitudes, falta de confianza o formación sobre la gripe o la VCG: "no pertenecer a un grupo de riesgo", "no haber padecido nunca la gripe", o "no confiar en la efectividad y/o seguridad de las vacunas", y podrían subsanarse mediante acciones de información y sensibilización específicas sobre este colectivo.

El absentismo laboral causado por la gripe estacional puede comprometer la asistencia en los centros sanitarios por falta de personal cuando es más necesario, estimándose que es responsable del 10-17\% de las bajas laborales en España, con una media de entre 5-7 días de duración y unas pérdidas anuales de 60 millones de horas de trabajo (23). El absentismo por gripe del 9,5\% referido por los FC en la temporada antigripal 2018-2019 es ligeramente inferior al 11,3\% (8) obtenido en el único estudio encontrado en nuestro país, realizado en la temporada 2010-2011, sobre todos los trabajadores de las farmacias de Lleida. Al igual que en este estudio, el absentismo laboral también fue menor entre los que se vacunaron que entre los que no, si bien esta relación no fue estadísticamente significativa. Según varias revisiones sistemáticas, los sanitarios vacunados pueden ver reducida la gripe de un 68-90\% (24) y los días de absentismo laboral entre el $28-40 \%$ (25).

Entre las estrategias dirigidas a aumentar la VCG a los grupos de riesgo, varios documentos proponen contar con la colaboración de los profesionales sanitarios en el diseño de las campañas de vacunación y que éstos aprovechen cualquier contacto de la población con el sistema sanitario para recomendar la vacunación $(3,5,6)$. En esta línea, prácticamente la totalidad de los FC (93,6\%) refirieron recomendar la VCG a la población, al igual que se ha evidenciado en otros estudios $(7,26,27,28)$. En ellos, esta recomendación sobre pacientes mayores de 65 años que no se habían vacunado, modificó la intención de no vacunarse en un 43,3\% (27) y un 29,4\% (26).

La disminución en España durante los últimos diez años de la cobertura vacunal contra la gripe en la población mayor o igual de 65 años, desde el 65,7\% (2009-2010) hasta el 54,2\% (2018-2019) (4), ha motivado considerar entre las estrategias para incrementarla, el estudio de la posibilidad de vacunar contra la gripe en farmacias comunitarias autorizadas. Esta medida ha demostrado aumentar con éxito dicha cobertura en varios países de dentro y fuera de Europa $(29,30,31,32)$. Este y otros estudios señalan que los FC estarian dispuestos mayoritariamente a administrar la VCG en la farmacia -si la Administración sanitaria lo autorizase y con una formación específica previa- lo que facilitaría la implementación de esta medida en nuestro país teniendo en cuenta la imprescindible coordinación con las administraciones sanitarias (33).

En conclusión, la información inédita proporcionada en este estudio muestra que la cobertura vacunal contra la gripe entre los FC españoles es más baja de lo deseable, en sintonía con el resto de profesionales sanitarios del SNS. El conocimiento de las causas para vacunarse y no vacunarse que refieren también aporta datos útiles para diseñar acciones de sensibilización específicas que incrementen directamente la cobertura vacunal en este colectivo e indirectamente en la población a la que atienden. Una de estas acciones sería facilitar la VCG de los FC, tal y como se efectúa en los centros sanitarios y algunas empresas. La gripe causa un absentismo relevante entre los FC. Hay una predisposición de los FC a participar en las estrategias de prevención de la gripe impulsando su papel como facilitador en la recomendación de la vacunación en pacientes de riesgo, y como colaborador en la administración de la VCG en farmacias comunitarias autorizadas.

\section{Limitaciones del estudio}

Este estudio, al igual que todos los basados en encuestas realizadas por correo, ya sea postal o electrónico, tiene una importante limitación, la baja participación y la voluntariedad de la misma que puede generar un sesgo de selección normalmente hacia los profesionales más motivados. Más aún porque coincidió con el inicio de la pandemia de COVID-19. No obstante, la participación de farmacéuticos de todas las comunidades autónomas y la ciudad autónoma de Melilla, tanto adjuntos como titulares, le da fortaleza al estudio.

Otra limitación, también frecuente en este tipo de estudios basados en encuestas, es que los datos son referidos por los participantes y no se consulta ningún registro. Esto facilita el sesgo de memoria. Además, la recomendación a la población a favor de la vacuna que refiere el farmacéutico es en general, y no sabemos si se realiza en el 100\% de las ocasiones de hacerla.

\section{Agradecimientos}

A la Sociedad Española de Farmacia Clínica, Familiar y Comunitaria (SEFAC) por su colaboración.

A todos los farmacéuticos comunitarios españoles que de forma anónima y altruista participaron en este estudio.

\section{Referencias bibliográficas}

1. Gripe e impacto de la vacuna antigripal. Temporada 2018-2019. Sistema de vigilancia de la gripe en España. Centro nacional de epidemiología. [Consultada el 9 de agosto de 2020]. Disponible en: http://vgripe.isciii.es/ inicio.do 
2. Consejo General de Colegios Oficiales de Farmacéuticos. Estadísticas de Colegiados y Farmacias Comunitarias 2019. Madrid: CGCOF; 2019. [Consultada el 9 de agosto de 2020]. Disponible en: https://statics-correofarmaceutico.uecdn.es/cms/sites/11/2019/06/ Estadisticas-de-Colegiados-y-Farmacias-2018.pdf

3. Comisión de Salud pública. Recomendaciones de vacunación frente a la gripe. Temporada 2020-2021. 5 de mayo de 2020. Consejo interterritorial del Sistema Nacional de Salud. [Consultada el 4 de agosto de 2020]. Disponible en: https://www.mscbs. gob.es/profesionales/saludPublica/ prevPromocion/vacunaciones/docs/ Recomendaciones_vacunacion_gripe. pdf

4. MSCBS [Internet]. Coberturas de vacunación frente a gripe en $\geq 65$ años, personas de 60-64 años, embarazadas y personal sanitario. Comunidades autónomas. Campaña 2018-2019. [Consultada el 9 de agosto de 2020]. Disponible en: https://www.mscbs. gob.es/profesionales/saludPublica/ prevPromocion/vacunaciones/calendario-y-coberturas/coberturas/home. htm

5. García A, Fernández Prada M, Aristegui J, Moreno D, Redondo E, Jimeno I, García Cenoz M, López Trigo JA. Documento de actualización y reflexión sobre la vacunación antigripal en España. Barcelona: Esmon Publicidad, S.A.; 2018. [Consultada el 4 de agosto de 2020]. Disponible en: https:// www.vacunas.org/wp-content/ uploads/2018/06/libro_gripe_v3_295-2018.pdf

6. Propuesta de Recomendación del Consejo sobre la intensificación de la cooperación contra las enfermedades evitables por vacunación. Diario Oficial de la Unión Europea C 466/2018 $n^{\circ} 61$, (28 de diciembre de 2018). [Consultada el 2 de agosto de 2020]. Disponible en: https://eur-lex.europa.eu/ legal-content/ES/TXT/HTML/?uri=CELEX:52018DC0244Etfrom=ES

7. Toledo D, Soldevila N, Guayta-Escolies R, Lozano P, Rius P, Gascón P, et al. Knowledge of and Attitudes to Influenza Vaccination among Community Pharmacists in Catalonia (Spain). 2013-2014 Season: A Cross Sectional Study. Int. J. Environ. Res. Public Health 2017; 14:756. doi:10.3390/ ijerph14070756

8. Nou C, Godoy P. Cobertura de vacunación antigripal de los trabajadores de oficinas de farmacia. Vacunas. 2012; 13 (4):145-149. doi:10.1016/ S1576-9887(12)70056-0

9. Grupo de trabajo de la Ponencia de Programa y Registro de Vacunaciones. Vacunación en trabajadores sanitarios. Madrid: Comisión de Salud Pública del Consejo Interterritorial del Sistema Nacional de Salud. Ministerio de Sanidad, Servicios Sociales e Igualdad; 2017.
[Consultada el 6 de agosto de 2020]. Disponible en: https://www.mscbs. gob.es/profesionales/saludPublica/prevPromocion/vacunaciones/vacunas/ docs/Vacunacion_sanitarios.pdf

10. MSSSI [Internet]. Coberturas de vacunación frente a gripe en $\geq 65$ años, personas de 60-64 años, embarazadas y personal sanitario. Comunidades autónomas. Campaña 2017-2018. [Consultada el 2 de agosto de 2020]. Disponible en: https://www.mscbs. gob.es/profesionales/saludPublica/ prevPromocion/vacunaciones/calendario-y-coberturas/coberturas/docs/ Todas_las_tablas2017.pdf

11. El gripómetro [Internet]. Sanofi Pasteur. 2018. [Acceso 7 de agosto de 2020]. Disponible en: https://gripometro.es/el-gripometro/

12. Ruiz AD, Frei CR, Barner JC, Carson JJ, Oramasionwu CU, Ruiz JL et al. Influenza vaccination rates among pharmacists. Journal of the American Pharmacists Association. 2010 Jan 1;50(4):517-522. doi:10.1331/JAPhA.2010.09146

13. Porras-Povedano M, Santacruz-Hamer V, Franco-Álvarez de Luna F. Oliva-Reina I. Cobertura de vacunación antigripal en trabajadores de un centro sanitario. Vacunas. 2015; 16 (2): 4450. doi:10.1016/j.vacun.2015.07.005

14. Torner N, Godoy P, Soldevila N, Toledo D, Rius C, Domínguez A. Estudio actitudes sobre vacunación antigripal en profesionales sanitarios de atención primaria de Cataluña. Vacunas. 2016; 48 (3): 192-199. doi:10.1016/j. aprim.2014.09.014

15. Navalón-Ramon E, Lombardi A, Macià-Rosell E, Martínez-Ribes I. Cobertura de vacunación antigripal entre profesionales sanitarios de una zona básica de salud. Vacunas. 2013; 17 (2): 41-46. doi:10.1016/j.vacun.2016.08.001

16. Pérez-Ciordia i, et al. Cobertura vacunal y factores que motivan la actitud de vacunación antigripal en profesionales sanitarios. Vacunas. 2017; 18 (1): 3-10. doi:10.1016/j.vacune.2016.11.002

17. Montserrat-Capdevila J, Godoy P, Marsal JR, Barbé-Illa F. Factores asociados a recibir la vacunación antigripal en profesionales de atención primaria. Gac Sanit. 2015;29(5):383386. doi:10.1016/j.gaceta.2015.02.004

18. Picazo J.J, et al. Consenso sobre la vacunación frente a la gripe en el personal sanitario. Rev Esp Quimioter 2012;25(3): 226-239 [Consultada el 7 de agosto de 2020]. Disponible en: http://www.seq.es/seq/02143429/26/3/picazo.pdf

19. Domínguez A, Godoy P, Castilla J, Soldevila N, Toledo D, Astray J, et al. (2013) Knowledge of and Attitudes to Influenza Vaccination in Healthy Primary Healthcare Workers in Spain, 2011-2012. PLoS ONE 8(11): e81200. doi:10.1371/journal. pone. 0081200
20. Muncunill M, Bosch $\mathrm{M}^{\mathrm{a}} \mathrm{A}$, Tamborero G, Fonseca M. Factores condicionantes de la vacunación antigripal en profesionales de atención primaria. Gac Sanit. 2011; 25(5):439-440. doi:10.1016/j.gaceta.2011.04.014

21. Rodríguez Coronado V, García de Blas F, Reverte Asuero C, Herraiz Cristóbal R, Álvarez Villalba M, del Cura González M.I. Motivos de los trabajadores sanitarios de atención primaria para no vacunarse contra la gripe. Vacunas. 2009; 10(2): 37-41. doi:10.1016/S15769887(09)72268-X

22. Bellia et al. Healthcare worker compliance with seasonal and pandemic influenza vaccination. Influenza and other Respiratory Viruses. 2013. 7(Suppl. 2): 97-104. doi:10.1111/irv.12088

23. Ligero Lopez E, Vico Garcerán B. La gripe: causa frecuente de incapacidad temporal en personal sanitario. Rev Asoc Esp Med Trab. 2015; 24 (1):9-16. [Consultada el 6 de agosto de 2020]. Disponible en: http:// www.aeemt.com/Revista_AEEMT_NF/ VOL_24_N01_2015_MAR/NO_Socios/ VOL_24_N01_2015_MAR_Original01. html

24. Burls A, Jordan R, Barton $\mathrm{P}$, Olowokure B, Wake B, Albon $\mathrm{E}$, et al. Vaccinating healthcare workers against influenza to protect the vulnerables it a good use of healthcare resources? A systematic review of the evidence and an economic evaluation. Vaccine. 2006; 24(19):4212-21. doi:10.1016/j.vaccine.2005.12.043

25. Demicheli V, Jefferson T, Rivetti D, Deeks J. Prevention and early treatment of influenza in healthy adults. Vaccine. 2000 Jan 6;18(1112):957-1030. doi:10.1016/s0264-410 x(99)00332-1

26. Andrés-Rodríguez NF, Mera-Gallego R, Piñeiro-Abad A, Acuña-Ferradanes A, Mera-Gallego I, García-Rodríguez P, Andrés-Iglesias JC, Fornos-Pérez JA. Vacunación antigripal en la farmacia comunitaria: opinión de pacientes y farmacéuticos. Farmacéuticos Comunitarios. 2018 Sep 28; 10(3):15-24. doi:10.5672/FC.2173-9218.(2018/ Vol10).003.03

27. Aparicio C, Climent MT, Baixauli VJ, Rodrigo MJ, Albanell F, Recio MC. Intervención del farmacéutico comunitario en la vacunación de adultos con patología respiratoria. Farmacéuticos Comunitarios. 2018 Sep 28; 10(3):514. doi:10.5672/FC.2173-9218.(2018/ Vol10).003.02

28. Lustenko V. et al. Vacunación antigripal en pacientes externos de riesgo. Impacto de la intervención farmacéutica. Rev. OFIL 2016; 26(4):275279. Disponible en: https://www. revistadelaofil.org/vacunacion-antigripal-pacientes-externos-riesgo-impacto-la-intervencion-farmaceutical 
29. Rosado H, Bates I. An overview of current pharmacy impact immunisation A global report. The Hague: International Pharmaceutical Federation (FIP); 2016. [Consultada el 2 de agosto de 2020]. Disponible en: https://www.fip.org/files/fip/publications/FIP_report_on_Immunisation.pdf

30. Isenor JE et al. Impact of pharmacists as immunizers on vaccination rates: A systematic review and me- ta-analysis. Vaccine (2016);34(47): 5708-5723. doi:10.1016/j.vaccine. 2016. 08.085

31. Kirkdale CL, Nebout G, Megerlin F, Thornley T. Benefits of pharmacist-led flu vaccination services in community pharmacy. Ann Pharm Fr. 2017 Jan;75(1):3-8. doi:10.1016/j. pharma.2016.08.005. Epub 2016 Oct 4. PMID: 27717412.

32. Czech et al. Flu Vaccinations in Pharmacies-A Review of Pharmacists
Fighting Pandemics and Infectious Diseases. Int. J. Environ. Res. Public Health 2020, 17, 7945. doi:10.3390/ ijerph17217945

33. Guayta-Escolies R, Urbiztondo L, Domínguez A, Bagaria G, Gascón P, de Dalmases J, et al. Coberturas vacunales y farmacia comunitaria: ¿un binomio estratégicamente necesario y operativamente factible? Vacunas. 2018;19:79-84. doi:10.1016/j.vacun. 2018.05.001 